\title{
Duale Bronchodilatation wird Kombination der ersten Wahl
}

\author{
Duale Bronchodilatation schützt bei schwerer COPD besser vor Exazerbationen als \\ die Kombination ICS/LABA, so das Ergebnis der direkten Vergleichsstudie FLAME. \\ Jetzt werden die Leitlinien umgeschrieben.
}

_ Die bisherigen GOLD-Leitlinien bei COPD sehen vor, dass in frühen Stadien mit einem LAMA (lang wirksamer Muskarin-Antagonist) oder einem LABA (lang wirksames Beta-2-Sympathomimetikum) und bei nicht ausreichender Symptomkontrolle mit einer LAMA/ LABA-Kombination behandelt wird. Bei hohem Risiko für Exazerbationen werden LABA/ICS (inhalatives Steroid) oder ein LAMA empfohlen.

\section{Meilensteinstudie FLAME}

Das wird sich mit den Ergebnissen der FLAME-Studie ändern. „Die Fixkombination zweier Bronchodilatatoren (LAMA plus LABA) wird die Kombination der ersten Wahl für alle COPD-Patienten werden; optimale Bronchodilatation wird der Grundbaustein der COPD-Therapie“, erklärte die renommierte Pneumologin Prof. Jadwiga Wedzicha vom National Heart and Lung Institute am London Imperial College,

Erstautorin der FLAME-Studie, bei einer offiziellen Pressekonferenz der American Thoracic Society in San Francisco.

Die doppelblinde FLAMEStudie hatte bei 3.362 schwerkranken COPD-Patienten (75\% im Stadium GOLD D) mit mindestens einer Exazerbation im Vorjahr prospektiv über 52 Wochen zwei Therapiestrategien miteinander verglichen: Duale Bronchodilatation mit Indacaterol/Glycopyrronium (Ultibro $1 \times 110 / 50 \mu \mathrm{g} / \mathrm{d}$ ) und Bronchodilatation plus Entzündungshemmung mit Salmeterol/Fluticacon. Primärer Endpunkt war die jährliche Exazerbationsrate.

\section{Bisheriger Therapiestandard verliert den Doppelbind-Vergleich deutlich}

Die LABA/LAMA-Kombination erwies sich dem bisherigen Therapiestandard nicht nur als nicht-unterlegen, sondern als rundherum überlegen. „Die Ergebnisse sind in allen Patientengruppen und über alle Endpunkte sehr konsistent, das Ausmaß der Überlegenheit beeindruckt", so Wedzicha.

Alle Exazerbationen der Patienten wurden sorgfältig erfasst und dokumentiert. Im Studienjahr erlitten die Patienten der Indacaterol/GlycopyrroniumGruppe im Schnitt 3,59 Exazerbationen und die Patienten der Salmeterol/Fluticacon-Gruppe im Schnitt 4,03 Exazerbationen. Die relative Risikoreduktion betrug $11 \%(\mathrm{p}=0,003)$. Die Zeit bis zur

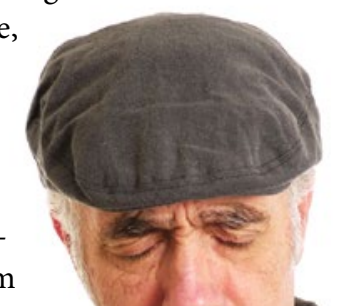

Duale Bronchodilatatoren können ihn vor Exazerbationen schützen. ersten Exazerbation wurde durch duale Bronchodilatation signifikant verlängert (71 vs. 51 Tage, Risikoreduktion um $16 \%$, $\mathrm{p}<0,001)$. Bei isolierter Betrachtung von moderaten bis schweren Exazerbationen betrug diese Risikoreduktion $22 \%$, bei Betrachtung nur schwerer Fälle mit Hospitalisation $19 \%$.

Die Risikoreduktion erwies sich als unabhängig von der Zahl der Eosinophilen im Blut, d.h. auch dieser Biomarker scheint untauglich, um Patienten zu identifizieren, die von einem ICS profitieren.

\section{Duale Bronchodilatation in allen Endpunkten signifikant überlegen} Auch in anderen Endpunkten schnitten die Patienten unter Indacaterol/Glycopyrronium signifikant besser ab: In der Lungenfunktion, beim Verbrauch von Notfallmedikamenten, in der Lebensqualität, bei der Inanspruchnahme des Gesundheitssystems. Patienten der ICS/ LABA-Gruppe erlitten signifikant mehr Pneumonien (4,8\% vs. $3,2 \%, \mathrm{p}=0.02)$.

Befragt nach den praktischen Konsequenzen erklärte Wedzicha, dass sie nun auch bei COPD-Patienten mit Exazerbationen primär zwei Bronchodilatatoren verschreibt. Nur wenn dies nicht ausreichend hilft, kann eine Triple-Therapie mit einem zusätzlichen inhalativen Steroid erwogen werden. COPD-Patienten, die mit ICS/LABA behandelt werden und darunter 1- bis 2-mal jährlich eine Exazerbation erleiden, würde sie auf eine LAMA/LABA-Kombination umstellen.

\section{Dr. Dirk Einecke}

- Quelle: American Thoracic Society International Conference, San Francisco 13.-18. Mai 2016; J.A. Wedzicha, et al. Indacaterol-Glycopyrronium versus Salmeterol-Fluticasone for COPD. NEngl J Med 2016; doi: 10.1056/NEJMoa1516385 